A ccording to the Skin Cancer Foundation, more than 3.5 million skin cancers and 76,000 melanomas are diagnosed each year in the United States, and, on average, one person dies from melanoma every hour. As with most diseases, the best way to fight melanoma is to prevent it. Unfortunately, the latest sunscreen ingredients that can help to reduce the risk of melanoma and other skin cancers have languished for decades awaiting approval from the US Food and Drug Administration (FDA).

The ultraviolet (UV) filters in sunscreen work by absorbing, reflecting or scattering the UV light emitted by the Sun. UVA radiation, which represents roughly $90 \%$ of UV radiation, can accelerate skin ageing, cause skin damage and create a risk of skin cancer by damaging DNA. The other component, UVB, leads to sunburn and also increases the risk of skin cancer. The most effective protection blocks both UVA and UVB. But ingredients delayed by the FDA approval process would provide additional options, especially for UVA protection.

The active ingredients used in sunscreens are regulated by the FDA as drugs. But the FDA has not approved an over-the-counter sunscreen ingredient since 1999. In 2002, it created a new pathway to market for non-prescription ingredients, such as sunscreens, that allowed manufacturers to use data from other countries to establish that a product is safe and effective. To qualify for this 'time and extent application' (TEA) process, the company must establish that a product is approved in at least one comparable country and that it has been in use for at least five years in sufficient quantity. The TEA process was designed to streamline the review of new ingredients, and the FDA said that it expected to complete the evaluation of sunscreen ingredients within 90-180 days.

\section{SLOW PROGRESS}

Unfortunately, it has not gone according to plan. After more than 12 years, the FDA has still not approved a single sunscreen ingredient through the TEA process. This means that Americans still lag behind the rest of the world regarding access to the latest UVA filters - even though these ingredients now have a long history of safe use in Europe, Australia and other parts of the world.

There are currently eight ingredients waiting for a decision from the FDA, some of which were submitted for approval as long ago as 2002. Bemotrizinol, for example, has been languishing in the TEA queue since 2005, despite being approved for use in the European Union (EU) in 2000.

In the past few months, some manufacturers have received letters in response to their applications, but for many this was the first feedback they had received. In the letters, the FDA consistently argues that the products must undergo additional safety testing.

The FDA seems to be backtracking on the TEA process. At a recent meeting of its Nonprescription Drugs Advisory Committee about pending sunscreen ingredients, the FDA argued that the approval in a comparable jurisdiction, such as the EU, and experience of safe marketing is insufficient to support the approval of a sunscreen ingredient in the United States. Rather, the FDA would like companies to perform additional safety testing unique to the United States. This might include studies of dermal safety, 'bioavailability', carcinogenicity, developmental and reproductive toxicity, and toxicokinetics. The FDA acknowledged that some of these tests would take at least two years.

The FDA's sluggish regulatory response prompted the formation of the Public Access to Sunscreens (PASS) Coalition in March 2013, for which I am a policy adviser. The coalition's mission is to work with the FDA, Congress, the White House, health providers and consumer organizations to establish a regulatory pathway for the timely and transparent pre-market review of new, safe and effective sunscreen ingredients. The coalition, which comprises cancer research organizations, academic scientists and sunscreen manufacturers among others, thinks that the FDA should ensure it is adopting a riskbased approach, taking into account the known risk of skin cancer and melanoma, and balancing the benefits of sunscreen protection against the potential risks. Additional testing should be required only if international experience, adverse event reporting, or other scientific information reveals that the product's risk profile demands it.

Efforts by PASS led to the introduction of the Sunscreen Innovation Act in March 2014. The act reforms the TEA process to establish a predictable and transparent process for the review of sunscreen ingredients to ensure that safe and effective products reach the market as soon as possible. It maintains the existing requirements for TEA products but ensures that the FDA's safety and effectiveness review is completed within statutory deadlines in a transparent way, including an opportunity for public comment. The act calls for a formal evaluation of the process and requires reports on the FDA's progress in processing applications to be made available to the public.

The bipartisan act passed the US Senate unanimously in September 2014 and the US House of Representatives unanimously in November 2014. It is expected to be signed by the President later this year.

The PASS coalition continues to fight for the enactment of the Sunscreen Innovation Act and to ensure that safe sunscreens reach the market as soon as possible. This provides a responsible solution to a problem that is exacerbating a public-health crisis. Giving Americans more choices and promoting sunscreen innovation will go a long way towards preventing a deadly disease.

Michael J. Werner is a partner with Holland and Knight in Washington DC and a policy adviser for the Public Access to Sunscreens Coalition. e-mail:michael.werner@hklaw.com 